\title{
A Mesh-Based Shape Analysis Framework
}

\author{
Jean-José Jacq ${ }^{1,2}$ and Christian Roux ${ }^{1,2}$ \\ ${ }^{1}$ Département Image et Traitement de l'Information \\ ENST Bretagne, Technopôle de Brest-Iroise, B.P. 832 - 29285 Brest - France \\ ${ }^{2}$ Laboratoire de Traitement de l'Information Médicale (ERM 0102 INSERM) \\ CHU Morvan, 5 Avenue Foch, 29200 Brest - France \\ \{Ju.Jacq, Christian.Roux\}@enst-bretagne.fr
}

\begin{abstract}
We propose an efficient and non-parametric hierarchical partitioning approach that operates on a general 3D shape. The method suggested makes it possible to process an arbitrary shape provided that it can be described as a triangle mesh. The basic idea is to extend the use of the basin districts concept on curved spaces - such a partitioning process being applied on valuation issued from the computation of main curvatures over a polyhedral support. Hierarchical construction of basin districts is obtained from a Watershed Transform. In our working case, an efficient use of this well known transformation implies to be able to manage front propagations on a polyhedral surface with speed controled by the local characteristics of surface geometry. Moreover, the ability to provide an intrinsic shape partition from any triangle mesh lead us to propose a robust extension to usual mesh simplification algorithms.
\end{abstract}

\section{Extended Summary}

The aim of this work is to provide an interactive, time efficient, flexible and unified set of cooperative shape partitioning tools. As the ultimate goal is to make it possible to an expert (physician, scientist, ...) to separate semi-interactively the major components of a shape, this work also refers to it as a shape dissection process. As a result, the common characteristic of the overall process we retained, is to work directly on triangle mesh without any global reparameterization. Although involving additional complexity, this generic approach offers two main advantages. On the one hand, as a triangle mesh is a shape representation commonly handled by any infographic system, the interactive use of the algorithms is guaranteed. On the other hand, processing without an intermediate reparameterization makes it possible to process complex biological shapes exhibiting an arbitrary topology.

Although innovating $[1,2,3]$, the main idea developed in this work is rather simple. If a Watershed Transform can be defined on a polyhedral support, then a global and hierarchical partition of the support can be performed. If these valuations are defined as functions of the local curvatures, the partition can be made intrinsic to the shape. Another key aspect of this shape partition approach is that it does not imply a very noise sensitive analysis through third order derivatives. Accordingly, it does not require the knowledge of the directions of the principal curvatures. So, the heart of this approach is mainly related to the extension of the immersion simulation [4] on unstructured meshes and involves, in particular, the ability to manage front propagations while solving the Eikonal equation on triangulated domains [5]. 
In order to get an operational framework, this working scheme requires a set of coprocessing tools operating directly on a triangulated domain. These tools are also studied here. The main requirement is to be able to manage common signal or image processing tasks (resampling, filtering, computing second order derivatives and geodesic distances) on a polyhedral support while presenting an optimal trade-off between accuracy and efficiency. This analysis framework is embedded in a standard 3-D infographic environment (OpenGL). Figure 1 depicts the full interactive framework when applied to analysis of articular surfaces of a fossil talus bone - this dissection case is based on torsion energy. The whole of the processing steps results depicted by Fig. 1, were obtained interactively from an Apple PowerBook G3.

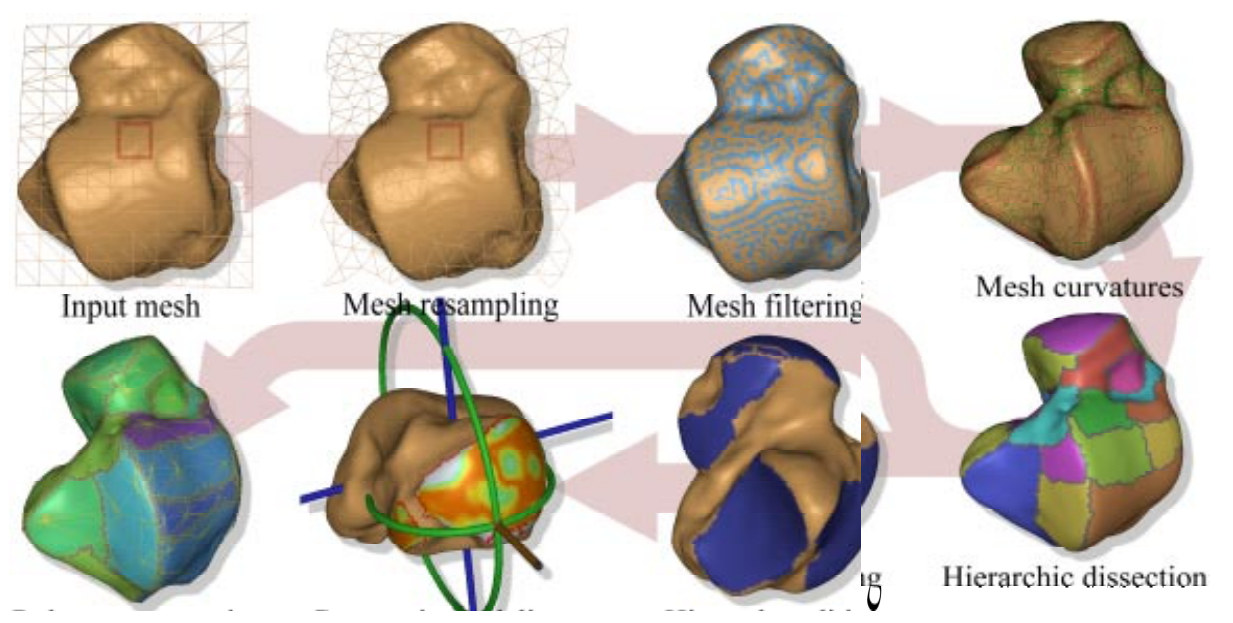

Fig. 1. A mesh-processing framework focusing on hierarchical dissection of anatomical shapes.

\section{References}

1. Jacq, J.J.: Partitionnement de Surfaces par Morphologie Géodésique. Application à la morphométrie Interactive du Complexe Péri-talien. In: Attelier Traitement et Analyse des Images, Méthodes et Applications, TAIMA'99, 23-26 Mars. 1999, Hammamet, Tunisie.

2. Jacq, J.J., Roux, C., Couture, C.: Segmentation morphologique géodésique de surfaces. Application à la morphométrie interactive des surfaces articulaires en anthropologie. In: 17 ième colloque GRETSI sur le Traitement du Signal et des Images, 13-17 Sept. 1999, Vannes., 39-42

3. Jacq, J.J., Roux, C.: Hierarchical Shape Dissection Through Front Propagation on Triangle Mesh with Applications. 2001 (Submitted)

4. Vincent, L., Soille, P.: Watersheds in Digital Spaces: an Efficient Algorithm Based on Immersion Simulations. IEEE Trans. on Pattern Analysis and Machine Intelligence, Vol. 13, No. 6, Jun. 1991, 583-598

5. Sethian, J.A.: Level Set Methods and Fast Marching Methods. Cambridge University Press, Aug. 1999, 378p

Acknowledgement. This work has been supported by a grant (99B406) from the French Administration of Research and Technology. 